\title{
QUARK JETS FROM ANTINEUTRINO INTERACTIONS (I). Net charge and factorization in the quark jets
}

\author{
J.P. BERGE, D. BOGERT, R. ENDORF', R. HANFT, J.A. MALKO, G.I. MOFFAT, \\ F.A. NEZRICK, R. ORAVA ${ }^{2}$ and J, WOLFSON \\ Fermi National Accelerator Laboratory, Batavia, Illinois 60510, USA
}

\begin{abstract}
V.V. AMMOSOV, A.G. DENISOV, G.S. GAPIENKO, V.A. GAPIENKO, V.I. KLUHKIN, V.I. KORESHEV, P.V. PITUKHIN, V.I. SIROTENKO, E.A. SLOBODYUK, Z.V. USUBOV and V.G. ZAETZ
\end{abstract}

Institute of High Energy Physics, Serpukhov, USSR

\section{V.I. EFREMENKO, A.V. FEDOTOV, P.A. GORICHEV, V.S. KAFTANOV, G.K. KLIGER, V.Z. KOLGANOV, S.P. KRUTCHININ, M.A. KUBANTSEV, I.V. MAKHLJUEVA, V.I.'SHEKELJAN and V.G. SHEVCHENKO \\ Institute for Theoretical and Experimental Physics, Moscow, USSR}

J. BELL, C.T. COFFIN, B.P. ROE, A.A. SEIDL, D. SINCLAIR and E. WANG

University of Michigan, Ann Arbor, Michigan 48104, USA

Received 11 September 1980

(Revised 3 December 1980)

We investigate properties of the hadron jets produced in deep inelastic antineutrino-nucleon charged current interactions in the Fermilab 15 foot bubble chamber. Our detailed analysis of the jet net charge provides evidence for the quark origin of the hadron jets. The factorization hypothesis for the inclusive one-particle cross section is found to be valid.

\section{Introduction}

Quark fragmentation is believed to be the origin of hadron jets observed in deep inelastic lepton-nucleon scattering [1], $\mathrm{e}^{+} \mathrm{e}^{-}$annihilation [2], and large transverse momentum hadron-hadron collisions [3].

In parton models the parton struck by the current in leptoproduction or produced in $\mathrm{e}^{+} \mathrm{e}^{-}$annihilation is thought to convert into the final-state hadrons having inclusive spectra which are independent of the initial state and which are determined

\footnotetext{
${ }^{1}$ Visitor from the University of Cincinnati, Cincinnati, Ohio.

${ }^{2}$ On leave from the University of Helsinki, Helsinki, Finland.
} 
only by the quark flavour and by the hadron fractional energy. Two characteristic features of hadron fragmentation in particle production in hadron-hadron interactions at high energy are the existence of a flat plateau in rapidity and the retention of hadron quantum numbers, on the average, in the hadron fragmentation region. Berman, Bjorken and Kogut have argued that parton fragmentation should also develop a plateau [4] and Feynman has suggested that the quantum numbers of the quark-parton are also retained, on the average, in the quark fragmentation region [5].

Properties of the jets are, at present energies, well described by the parametrization of Field and Feynman who assume a simple momentum sharing in the quark fragmentation process, the size of $\mathrm{SU}(3)$ symmetry violation, and the spin nature and the limited transverse momentum of the primary mesons [6]. Many of the experimental tests have been, however, insensitive to the basic hypothesis that quark fragmentation is the origin of the observed hadrons [7].

It is the purpose of this study to find evidence for the quark origin of the hadron jets in deep inelastic leptoproduction. In this paper we will particularly concentrate on the net charge and factorization properties of the inclusive particle spectra of the hadrons produced in antineutrino-nucleon charged current interactions. In two forthcoming papers, we will extend our analysis to inclusive single-particle distributions, particle ratios and multiplicities in the jets, and the transverse structure of the antineutrino charged current induced jets.

\section{Experimental details}

The experimental analysis is based on events photographed in the Fermilab 15 foot bubble chamber filled with a neon-hydrogen mixture (64\% atomic neon) exposed in a first run to a double horn focused wide-band antineutrino beam [8] and in a second run to a bare target sign selected antineutrino beam [9]. In the first run an absorptive plug suppressed the neutrino flux. The neutrino component in our antineutrino event sample is about $15 \%$, averaged over the antineutrino energy spectrum for antineutrino energy $E_{\bar{\nu}}>10 \mathrm{GeV}$.

A $\bar{\nu}_{\mu} \mathrm{N}$ charged current interaction produces hadrons and a positively charged muon which balances the total transverse momentum of the hadrons. The muon is identified by the External Muon Identifier [10] supplemented by a large transverse momentum procedure (BIGPT). The EMI's efficiency is the product of a geometric acceptance and an instrumental efficiency which is approximately flat as a function of muon momentum $p_{\mu}$ for $p_{\mu}>10 \mathrm{GeV} / c$. The BIGPT muon identification procedure identifies the muon candidate by testing the transverse momentum of the candidate $\left(p_{\mathrm{t}}\right)$ with respect to the direction of the visible momentum of the observed hadrons as indicated in fig. 1. Using events containing EMI identified muons we demonstrate in fig. 1 that charged hadrons have $p_{\mathrm{t}}$ well below $1.6 \mathrm{GeV} / c$ but the muons generally have much larger $p_{\mathrm{t}}$. This and similar studies suggested the following algorithm: when the charged track with the largest $p_{\mathrm{t}}$ in the event is found, 


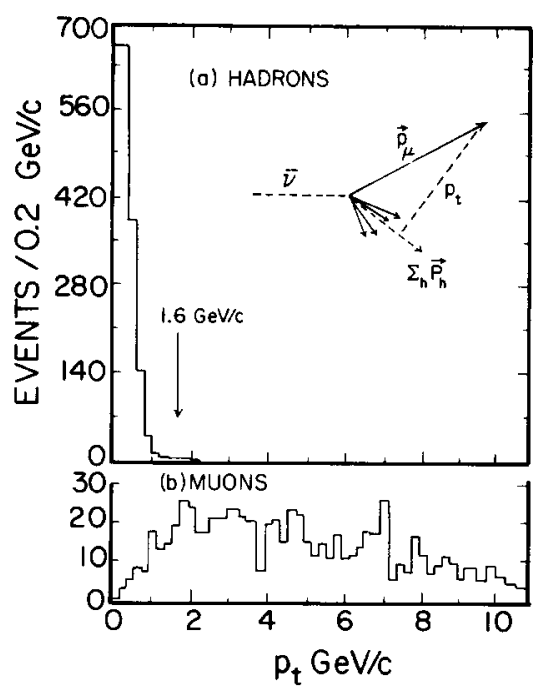

Fig. 1. Definition of the variable $p_{t}$ discussed in the text and the $p_{1}$ distributions for (a) the final-state hadrons and (b) EMI-identified muons.

it is selected as a muon if it has either (i) $p_{\mathrm{t}}>1.6 \mathrm{GeV} / c$ or (ii) $p_{\mu}>4 \mathrm{GeV} / c$ and angle relative to the beam direction greater than 0.2 radians. Our studies show that the BIGPT selected muon sample contains less than $2 \%$ hadron contamination. As can be seen from fig. 2, BIGPT is significantly more efficient than the EMI when $p_{\mu}>10 \mathrm{GeV} / c$, but low momentum muons $\left(p_{\mu}<10 \mathrm{GeV} / c\right)$ are more efficiently identified by EMI [11]. The two identification methods are complementary; the overall $\mu^{+}\left(\mu^{-}\right)$identification efficiency is $92 \%(87 \%)$ independent of muon production angle for muons having $p_{\mu}>4 \mathrm{GeV} / c$.

Charged hadron tracks not giving a unique mass fit in a geometry program were treated as pions. Special attention was given to the determination of the charge and momentum of the hadron tracks which were difficult to measure accurately. Hadrons which interacted or decayed in too short a distance in the bubble chamber to allow an adequate momentum estimate or charge determination were analyzed separately. For these tracks the momentum and charge were estimated from the tracks emerging from the secondary interaction or decay. If the relative error in this estimated momentum, $\Delta p / p$, exceeded $40 \%$, the whole event was rejected $(8 \%$ of the total sample of events). As an alternative procedure, all tracks having relatively large $\Delta p / p$ were rejected (7\% of all tracks), and momentum-dependent weights were assigned to all tracks to account for the close-in interactions. The average weight used in this procedure was 1.09. Resulting inclusive spectra from the two procedures were found to be in a good agreement.

Scanning efficiencies, ranging from $65 \%$ for two-pronged events to $99 \%$ for sixand higher-pronged events, were accounted for by assigning multiplicity dependent weights to all events. 

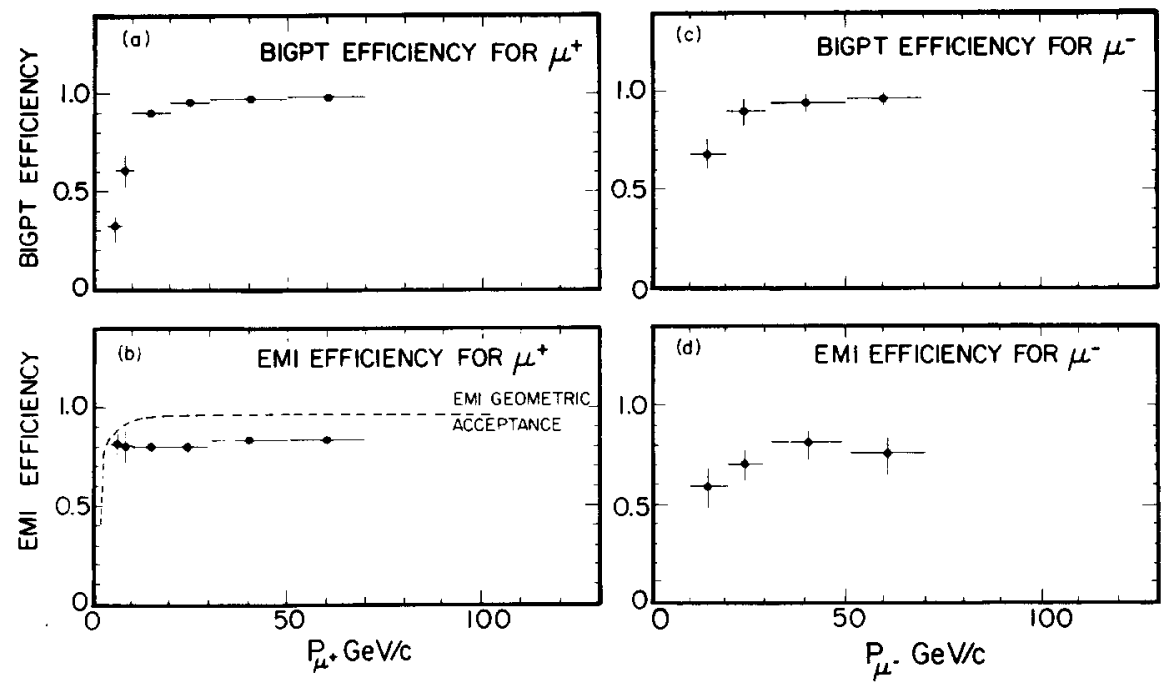

Fig. 2. Muon identification efficiencies using the external muon identifier (EMI) and the kinematical method (BIGPT) (see the text) for ( $a$ and $b$ ) the positively charged muons and ( $c$ and $d$ ) the negatively charged muons as a function of the muon momentum. The efficiencies are evaluated by considering the two methods as independent detectors.

The antineutrino (neutrino) energy spectrum peaks at $18 \mathrm{GeV}(26 \mathrm{GeV})$ and extends up to $200 \mathrm{GeV}$ (fig. 3). On the average $17 \%$ of the hadronic energy escapes detection in the bubble chamber. The analysis was performed using several energy correction procedures, for example, event-by-event methods [12, 13], and an average energy correction method [11]. The physics results presented here were found to be insensitive to the method employed and are actually based on the method of ref. [13].

Energy smearing in inclusive distributions is taken into account by evaluating energy desmearing functions for each inclusive spectrum from a Monte Carlo program [7] which was suitably modified to meet our experimental conditions. In this Monte Carlo model the hadronic final states are generated, for a given hadronic c.m. energy $(W)$, according to longitudinal phase space for produced mesons plus an approximately flat c.m. distribution in Feynman $x\left(x_{\mathrm{F}}\right)$ for the recoiling nucleon in the interval $-0.95<x_{\mathrm{F}}<0$. The Monte Carlo events are constrained to conserve energy, charge and momentum, but there are no particle-particle correlations built in. The numbers of negatively charged pions produced are constrained to follow the parametrization $\left\langle n^{-}\right\rangle=-0.25+0.76 \ln W^{2}$ evaluated for our data.

Finally, the antineutrino (neutrino) event sample was required to have (a) a positively (negatively) charged muon with momentum greater than $4 \mathrm{GeV} / c$ and (b) a total momentum along the antineutrino (neutrino) direction larger than 7.5 $\mathrm{GeV} / c$. The charged current antineutrino (neutrino) event sample passing these selection criteria consists of 7200 (1100) unweighted events. 

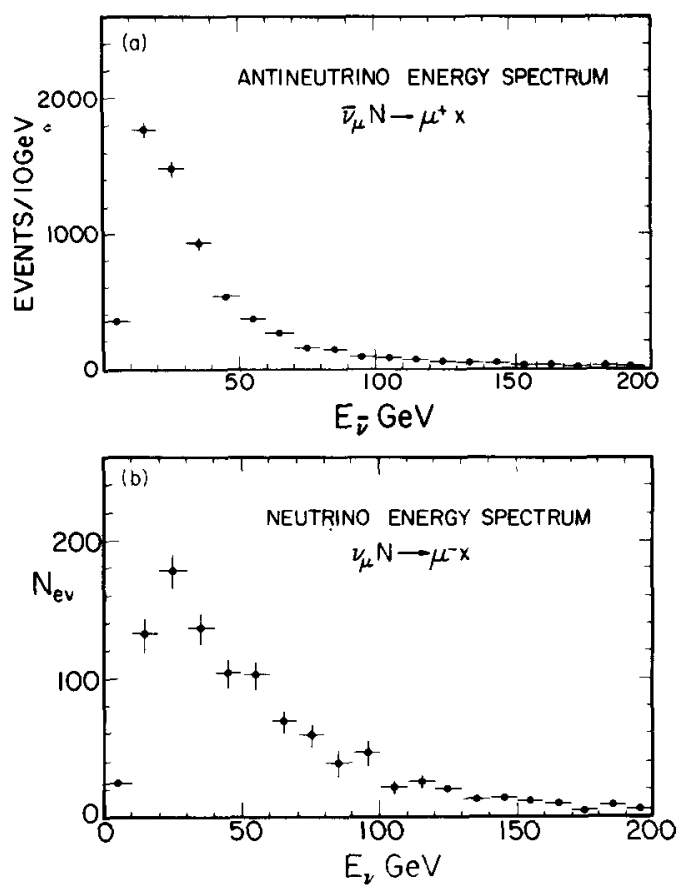

Fig. 3. (a) Antineutrino energy spectrum and (b) neutrino energy spectrum as obtained from the reconstructed event energies.

\section{Jet definition}

There are several ways to define the current jet. Based on the limited transverse momentum of the secondary hadrons relative to the current direction one can define a cylinder which contains most of the current jet or one can choose an angular cone relative to the current direction to include the hadrons belonging to the current jet. However, for our inclusive analysis we will base our selection of the current jet on the rapidity distribution of the net charge of the final-state hadrons as seen in the hadronic c.m.s.

Detailed consideration of the rapidity distributions of the hadrons resulting from deep inelastic interactions is given by Bjorken [14]. He separates the following distinct rapidity regions (fig. 4):

(i) Particles moving rapidly forward in the current direction in the hadron c.m.s. arise from the fragmentation of a quark which the current has knocked out of the target nucleon (region $V$ in fig. 4).

(ii) Particles moving to the opposite, backward, direction relative to the current direction in the hadron c.m.s. lie in the target fragmentation region and arise from the "hadronization" of the partons which remained after the collision (region 1).

(iii) Particles moving slowly with respect to the c.m. lie in the central region (target plateau, region II, and current plateau, region IV). Their distribution is flat in 


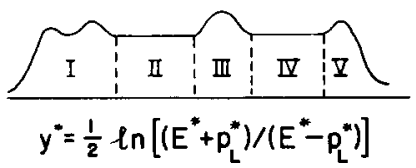

Fig. 4. Rapidity distribution of the hadrons resulting from the quark fragmentation process divided into: target fragmentation region (I), target plateau (II), hole fragmentation region (III), current plateau (IV), and quark fragmentation region (V).

rapidity. The central region also contains the region of phase space formerly occupied by the struck quark before it was removed (the hole fragmentation region, region III).

The rapidity distributions of charged hadrons produced in the antineutrino-nucleon charged current interactions in this experiment are shown in fig. 5 in three different c.m. energy intervals. The rapidity in the c.m.s. is $y^{*}=\frac{1}{2} \ln \left(\left(E^{*}+p_{\mathrm{L}}^{*}\right) /\left(E^{*}-p_{\mathrm{L}}^{*}\right)\right)$, where $E^{*}$ is the hadron c.m. energy and $p_{\mathrm{L}}^{*}$ the hadron c.m. momentum along the current direction. The length of the total rapidity interval is proportional to $\ln W^{2}$, while the length of the current fragmentation region is proportional to $\ln \left(-q^{2}\right)$, where $-q^{2}$ is the current mass squared. Hence, one should select relatively large values of $W$ to ensure adequate separation of the target and current fragments. It is conjectured that the hole fragmentation region should be observed only at very small Bjorken $x$ values [14] $\left(x_{\mathrm{B}}=-q^{2} / 2 M \nu\right.$, where $M$ is the nucleon mass and $\nu$ the current energy), $x_{B} \simeq 10^{-3}$. No structure which could be attributed to the hole

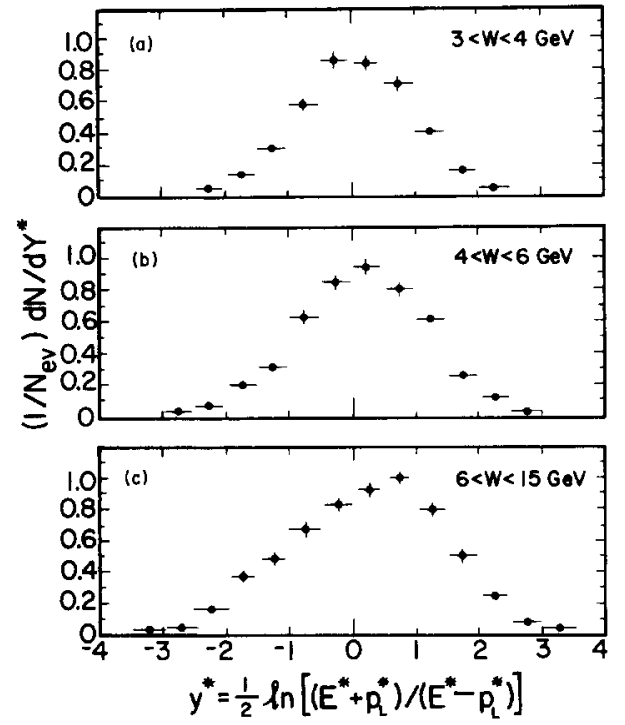

Fig. 5. Rapidity distributions of charged hadrons produced in antineutrino-nucleon charged current interactions in three separate c.m. energy intervals: (a) $3<W<4 \mathrm{GeV}$, (b) $4<W<6 \mathrm{GeV}$ and (c) $6<W<15 \mathrm{GeV}$. 
fragmentation region in the rapidity distribution of charged hadrons produced in our sample of antineutrino-nucleon charged current interactions is observed in the $x_{\mathrm{B}}$ range accessible to this experiment $\left(x_{\mathrm{B}}>0.01\right)$.

The net charge distribution of the hadrons, $\left(1 / N_{\mathrm{ev}}\right) \mathrm{d}\left(N^{+}-N^{-}\right) / \mathrm{d} y^{*}$, where $N_{\mathrm{ev}}$ and $N^{ \pm}$are the numbers of events and charged tracks, respectively, can be used to show that in the hadron c.m.s. the current jet is most readily separated [15]. As variables for the inclusive distributions, we use either the c.m. rapidity $y^{*}$, or the fractional energy in the laboratory system $\left(z=E_{\mathrm{h}} / \nu\right.$, where $E_{\mathrm{h}}$ is the hadron energy). To define the current jet, we transform the hadron four vectors into the hadronic c.m.s. and require that the c.m. rapidity of each hadron is positive, i.e., $y^{*}>0$.

The following phenomena affect the jet definition: (a) the intrinsic transverse momentum of the initial quark, which contributes to the overlap between the target and current fragments in the region $-0.2<x_{\mathrm{F}}<+0.2$ (ref. [16]), where $x_{\mathrm{F}} \simeq$ $2 p_{\mathrm{L}}^{*} / W$; (b) target mass effects, which can be neglected for the selection $-q^{2}>1$ $\mathrm{GeV}^{2} / c^{2}$ (ref. [16]), and (c) the energy reconstruction procedure, which results in a $2-15 \%$ smearing correction, depending on $x_{F}$, in the inclusive spectra of the final-state hadrons.

The antineutrino (neutrino) charged current induced jets are predicted to be dominantly d-quark (u-quark) jets, but some mixture of s-quark jets from the Cabibbo-suppressed transition $\mathrm{u} \rightarrow \mathrm{s}$ and $\overline{\mathrm{u}}$-quark ( $\overline{\mathrm{d}}$-quark) jets from the interactions off sea quarks in the target nucleon, $\overline{\mathrm{d}} \rightarrow \overline{\mathrm{u}}(\overline{\mathrm{u}} \rightarrow \overline{\mathrm{d}})$ is expected. The relative amount of s-quark jets is proportional to $\tan ^{2} \theta_{\mathrm{C}} \simeq 0.04$, where $\theta_{\mathrm{C}}$ is the Cabibbo angle. Our sea-quark density distribution is shown in fig. 6 . At $x_{\mathrm{B}} \leqslant 0.05$ there is about $70 \%$ contribution from antiquarks in the sea but above $x_{\mathrm{B}}=0.1$ this contribution is only $6 \%$. In the following we shall select $x_{\mathrm{B}}>0.1$ to investigate the predictions for the d-quark (u-quark) jets.

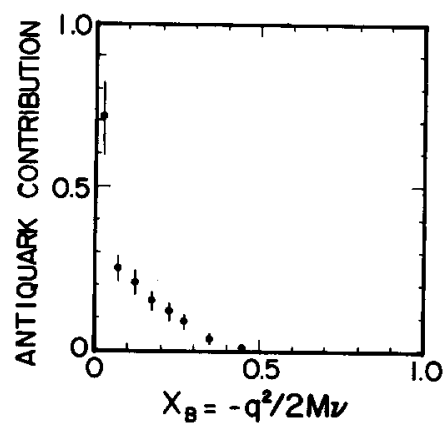

Fig. 6. Relative amount of antiquarks in the nucleon as a function Bjorken $x:\left(\bar{q}\left(x_{\mathrm{B}}\right)+2 \bar{s}\left(x_{\mathrm{B}}\right)\right) /\left(q\left(x_{\mathrm{B}}\right)\right.$ $\left.+\bar{q}\left(x_{\mathrm{B}}\right)+2 s\left(x_{\mathrm{B}}\right)+2 c\left(x_{\mathrm{B}}\right)\right)$, where $q\left(x_{\mathrm{B}}\right)\left(\bar{q}\left(x_{\mathrm{B}}\right)\right), s\left(x_{\mathrm{B}}\right)\left(\bar{s}\left(x_{\mathrm{B}}\right)\right)$ and $c\left(x_{\mathrm{B}}\right)$ represent non-strange, strange and charmed quark (antiquark) density distributions in the nucleon. The result is obtained by measuring the shape parameter of the $y$-distribution, $B^{\bar{\nu}}$, as a function of $x_{\mathrm{B}}$ and then evaluating $\frac{1}{2}\left(1-B^{\bar{\nu}}\right)$ which measures the relative amount of antiquarks in the nucleon. 


\section{Jet net charge}

In the quark-parton picture of quark fragmentation, quantum numbers of the fragmenting quark are retained, on the average, in the quark jet. Any selection for the current fragments $\left(y^{*}>y_{0}\right)$ must be made, however, to the final-state hadrons observed in the bubble chamber and not to the individual quarks as required by the hypothesis of exact retention of the quark quantum numbers. A selection $y^{*}>y_{0}$ can be made for any c.m. rapidity $y_{0} \geqslant 0$; we choose $y_{0}=0$. Fig. 7 illustrates the experimental selection procedure of the current fragments. The selection procedure $y^{*}>y_{0}$ necessarily "cuts" some of the quark-antiquark lines and leaves some of the quarks out of the selected region. Thus, a "leakage" of one quark results if a meson ( $\mathrm{q} \overline{\mathrm{q}}$ pair) is produced with a rapidity below the selection $y^{*}>y_{0}$ [17]. In the case of baryon production (qqq) the selection procedure "cuts" two quark-antiquark lines and a "leakage" of a combination of two quarks results [18] (fig. 7). Although this idea of quark quantum number retention-modulo the "leakage" factor-is not a characteristic of all theoretical models, it has been shown that the space-time structure of the fragmentation process selects the models containing the quantum number retention [19].

Let $p_{\mathrm{u}}, p_{\mathrm{d}}$ and $p_{\mathrm{s}}$ be the probabilities of finding a quark with flavor $\mathrm{u}, \mathrm{d}$ or $\mathrm{s}$ in the quark jet cascade. Neglecting other quark flavours, probability conservation gives $p_{\mathrm{u}}+p_{\mathrm{d}}+p_{\mathrm{s}}=1$. Isospin symmetry requires $p_{\mathrm{u}}=p_{\mathrm{d}}=p$ and thus $2 p+p_{\mathrm{s}}=1$. The mean of any additive quantum number of the hadrons in the current jet $(\langle N\rangle)$ can then be expressed as a sum of the original quark quantum number $\left(N_{\mathrm{q}}\right)$ and the leakage term $\left(L_{N}\right)$ which corresponds to one average quark or an average of all the relevant two-quark combinations, i.e., $\langle N\rangle=N_{\mathrm{q}}-L_{N}$, where $L_{N}=\alpha \Sigma_{i} p_{i} N_{i}+(1-$ $\alpha) \sum_{i j} p_{i} p_{j} N_{i j}$ ( $\alpha$ is the relative amount of mesons at the selection $y^{*} \simeq y_{0}$ ). The baryons contribute qq pairs to the leakage only when they are produced at $y^{*} \simeq y_{0}$. A proton contamination of $\left(15^{+} 3\right) \%$ (sect. 5) exists in our sample of positively charged mesons. We have estimated that this contamination $(1-\alpha=0.15)$ could produce about $5 \%$ maximum decrease of the leakage term $L_{N}$ if all the unidentified protons were produced at $y^{*} \simeq y_{0}$. In the following, we shall neglect the baryon term in the definition of $L_{N}$ and simply take $L_{N}=\Sigma_{i} p_{i} N_{i}$ (i.e., we take $1-\alpha=0$ ). We

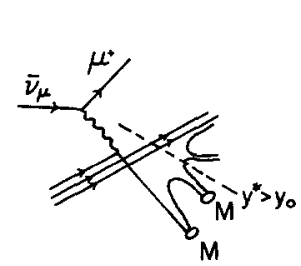

(a)

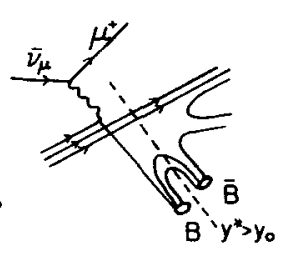

(b)

Fig. 7. Schematic illustration of the origin of the quantum number leakage in (a) meson production and (b) in baryon production. The dashed line represents a physical selection of the current fragments. 
then get, for example, for the isospin $\left\langle I_{z}\right\rangle=I_{\mathrm{q}}-L_{I}=I_{\mathrm{q}}$; for strangeness $\langle S\rangle=S_{\mathrm{q}}$ $-L_{S}=p_{\mathrm{s}}$ for the u- and d-quark jets, and $\langle S\rangle=-\left(1-p_{\mathrm{s}}\right)$ for the s-quark jets; for baryon number $\langle B\rangle=B_{\mathrm{q}}-L_{B}=\frac{1}{3}-\frac{1}{3} \Sigma_{i} p_{i}=0$; and for the jet net charge $\langle Q\rangle=Q_{\mathrm{q}}$ $-L_{Q}=Q_{\mathrm{q}}-p Q_{\mathrm{u}}-p Q_{\mathrm{d}}-p_{\mathrm{s}} Q_{\mathrm{s}}=1-p$ for the u-quark jets, and $\langle Q\rangle=-p$ for the $\mathrm{d}-$ and s-quark jets.

Rapidity distributions of the net charge of the hadrons produced in antineutrinonucleon charged current interactions are shown in fig. 8 for three different $W$ intervals. Separation of the current jet is observed as the decreasing net charge in the central region. At finite energies, however, there is always an overlap between the target and current fragmentation regions which diminishes as $W$ increases. The area lost from the current jet net charge distributions can be estimated by properly parametrizing the tail of the net charge distribution through the overlap. Assuming that the particle-particle correlations in the central region are of short-range order, we are led to a parametrization $\left(1 / N_{\mathrm{ev}}\right) \mathrm{d}\left(N^{+}-N^{-}\right) / \mathrm{d} y^{*}=C \exp \left(-\lambda \Delta y^{*}\right)$, where the parameter $\lambda$ is related to the correlation length in the central region [20] and $\Delta y^{*}=\left|y^{*}-y_{\max }^{*}\right|$. Since the maximum rapidity interval available is proportional to $\ln W^{2}$, the contribution from the overlap is proportional to $W^{-2 \lambda}$.

We have estimated $\lambda=0.5 \pm 0.1$ from our data by plotting $\ln \langle Q\rangle$ versus $\ln W$. We note further that the rapidity distributions for the net charge in the highest energy $\mathrm{pp}$ and $\pi \mathrm{p}$ experiments are well parametrized with an exponential in the central rapidity region, $\left(1 / N_{\mathrm{ev}}\right) \mathrm{d}\left(N^{+}-N^{-}\right) / \mathrm{d} y^{*}=C \exp \left(-0.54 \Delta y^{*}\right)$ [21]. This result $(\lambda$ $=0.54)$ indicates similar particle-particle correlation lengths in the $\mathrm{pp}$ and $\pi \mathrm{p}$ final

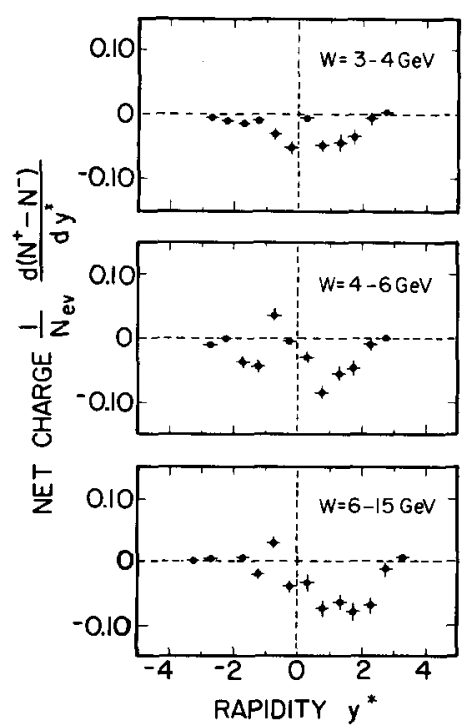

Fig. 8. The net charge of the hadrons produced in $\bar{\nu}_{\mu} \mathrm{N}$ charged current interactions as a function of the c.m. rapidity in three different $W$-intervals: $3<W<4 \mathrm{GeV}, 4<W<6 \mathrm{GeV}$, and $6<W<15 \mathrm{GeV}$. 


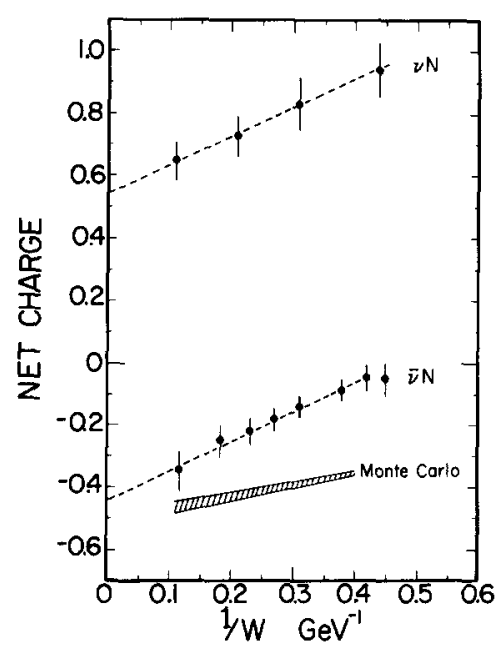

Fig. 9. Average net charge of the hadrons traveling forward in the hadronic c.m.s. as a function of $W^{-1}$. The dashed line represents a linear fit to the data points above $W=3 \mathrm{GeV}$. The shaded area is a prediction obtained from the Monte Carlo program which does not include the hypothesis of quark fragmentation.

states and our antineutrino-induced hadron jets in the central rapidity region. In fig. 9, we show the jet net charge as a function of $W^{-1}$ for our antineutrino and our neutrino charged current events. Extrapolation of the jet net charge to infinite $W$ gives $\langle Q\rangle^{\bar{\nu}}=-(0.44 \pm 0.09)$ and $\langle Q\rangle^{\nu}=0.54 \pm 0.12$ for the antineutrino and neutrino charged current events, respectively. As shown in fig. 9, the prediction of our Monte Carlo model, which does not include the assumption of quark fragmentation, does not give the same $W$-dependence for the average net charge as our antineutrino data $^{\star}$.

Our result for the overlap-free jet net charge in the antineutrino (neutrino) charged current induced jets measures the relative probability, $p$, of finding either a $\mathrm{u}$ - or d-quark in the quark jet cascade [1]: $\langle Q\rangle^{\bar{\nu}}=-p\left(\langle Q\rangle^{\nu}=1-p\right)$. This probability gives for the $\mathrm{SU}(3)$ symmetry violation in the formation of q $\overline{\mathrm{q}}$ pairs $p_{\mathrm{s}} / p=$ $0.27_{-0.27}^{+0.40}$. In the same experiment, we have used the $\mathrm{K}^{0} / \pi^{-}$ratio in the antineutrino charged current induced jets to obtain $p_{\mathrm{s}} / p=0.27 \pm 0.04$ (ref. [22]). One should note that exact $\mathrm{SU}(3)$ symmetry would imply $p_{\mathrm{s}} / p=1$. Combining this result with the above net charge result we get $p=0.44 \pm 0.05$. For the average strangeness either in the u- or d-quark jets, this combined value of $p$ gives $\langle S\rangle=0.12 \pm 0.10$. The $\mathrm{K}^{0} / \pi^{-}$ratio alone as measured in this experiment would give $p=0.44 \pm 0.01$ and $\langle S\rangle=0.12 \pm 0.02$, respectively.

We compare our results with other estimates of the SU(3) symmetry violation in the quark jets obtained from published results of proton-proton and leptoproduction

* The fact that a relative amount of $\bar{\nu}$-sea quark interactions increases as $W$ increases has a marginal effect on the net charge extrapolation result as can be seen by excluding the highest $W$ point when fitting the straight line through the measured points. 
experiments. From the $\mathrm{K}^{+} / \pi^{+}$ratio in high energy proton-proton experiments [23] extrapolated to the Feynman $x$ of one (to avoid resonance contributions), we estimate $p_{\mathrm{s}} / p \sim 0.50$. Another estimate of $p_{\mathrm{s}} / p$ can be obtained from the cross section ratios $\left(\mathrm{J} / \psi \rightarrow \mathrm{K}^{+} \mathrm{K}^{*}\right) /(\mathrm{J} / \psi \rightarrow \rho \pi)$ corrected for phase-space factors [24]. The result $p_{\mathrm{s}} / p=0.49 \pm 0.11$ implies $p=0.40 \pm 0.02$. An electroproduction experiment obtains for the ratio $\left(\mathrm{K}^{0}+\overline{\mathbf{K}}^{0}\right) /\left(\pi^{+}+\pi^{-}\right)$a value of $0.13 \pm 0.03$ which the authors interpret as the ratio $p_{\mathrm{s}} / p$ (ref. [25]); this value would mean considerably stronger SU(3) symmetry violation in the quark jets. A jet net charge measurement in the same experiment, on the other hand, gives $p_{\mathrm{s}} / p=0.36$ (ref. [26]), which is again consistent with our measurements.

Field and Feynman have proposed an alternative way of distinguishing quark jets of different flavour [6]. There, one weights each particle with a $z$-dependent weight such that particles closer to the overlap region get a small weight and particles with large fractional energy $z$ (further from the overlap region) get a large weight; i.e., the weighted charge is defined as $Q_{\mathrm{w}}^{\nu, \bar{\nu}}=\Sigma_{i}\left(z_{i}\right)^{r} e_{i}$, where $r$ is a small number and $e_{i}$ is the integer charge of the $i$ th hadron in the final state. Resulting distributions from our experiment are shown in fig. 10 (fig. 11) for antineutrino (neutrino) charged current

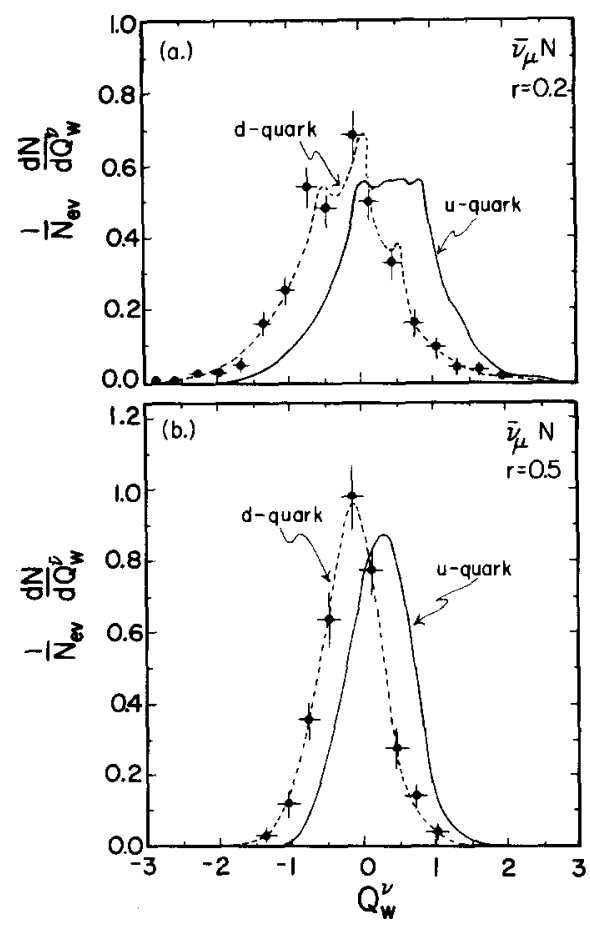

Fig. 10. Weighted charge $Q_{\mathrm{w}}^{\bar{v}}=\Sigma_{i}\left(z_{i}\right)^{r} e_{i}$ for the antineutrino charged current induced hadrons traveling forward in the hadronic c.m.s. (a) for $r=0.2$, and (b) for $r=0.5$. The solid curves represent the Field and Feynman predictions for the hadrons arising from the fragmentation of a u-quark with $10 \mathrm{GeV} / \mathrm{c}$ incident momentum and the dashed lines the corresponding predictions for the $10 \mathrm{GeV} / c \mathrm{~d}$-quark jets. 


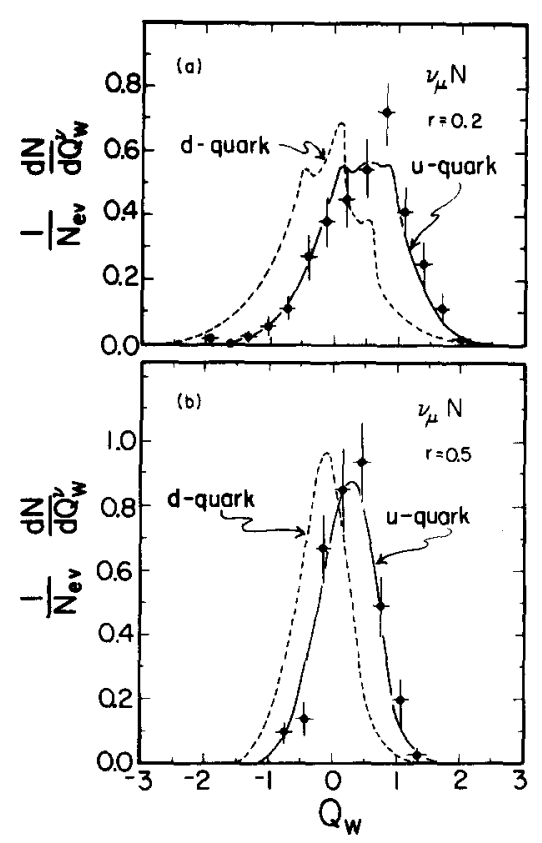

Fig. 11. Weighted charge $Q_{\mathrm{w}}^{\nu}=\Sigma_{i}\left(z_{i}\right)^{r} e_{i}$ for the neutrino charged current induced hadrons traveling forward in the hadronic c.m.s. (a) for $r=0.2$, and (b) for $r=0.5$. The solid curves represent the Field and Feynman predictions for the $10 \mathrm{GeV} / c$ u-quark jets and the dashed lines the corresponding predictions for the $10 \mathrm{GeV} / c \mathrm{~d}$-quark jets.

events. To compare with the predictions which are calculated for $10 \mathrm{GeV}$ quark jets, we select c.m. energies above $6 \mathrm{GeV}$. Corresponding predictions by Field and Feynman are shown for the d- and u-quark jets with the two values of $r, r=0.2$ and $r=0.5$ [6]. It is important to recognize that even though the Field and Feynman approach involves a parametrization of (other) leptoproduction data it gives predictions for the weighted charge which differ according to the flavour of the fragmenting quark. The average weighted charge values are given in table 1 with the predictions. Experimental results for the weighted charge for antineutrino (neutrino) charged current events are consistent with the predictions for the d-quark (u-quark) jets but not with the predictions for the u-quark (d-quark) jets.

We have considered possible effects caused by the use of a nuclear target in this experiment. Nuclear break-up products generally increase the visible net charge of the observed final state hadrons. Our selection criteria for the current fragments usually removes the slow secondary particles arising from the nuclear break-up, but it is expected that a small contamination from the nuclear fragments remains in our sample of events. To study these effects, we have selected a sample of events in which the net visible charge of the final state hadrons, $Q_{\mathrm{V}}$, corresponds to the initial state charge within one unit, i.e., we select $-2<Q_{\mathrm{V}}<1$. Effects of this selection on the measured jet net charge and on the measured weighted charge are summarized in 
TABLE 1

Average net charge $\langle Q\rangle^{\bar{\nu}},\langle Q\rangle^{\nu}$ and average weighted charge $\left\langle Q_{\mathrm{w}}\right\rangle^{\bar{\nu}},\left\langle Q_{\mathrm{w}}\right\rangle^{\nu}$ of the hadrons traveling forward in the hadronic c.m.s. in antineutrino and neutrino-nucleon charged current interactions

\begin{tabular}{lccc}
\hline Antineutrinos & $\langle Q\rangle^{\bar{\nu}}$ & $\left\langle Q_{\mathrm{w}}\right\rangle^{\bar{\nu}} r=0.2$ & $\left\langle Q_{\mathrm{w}}\right\rangle^{\bar{\nu}} r=0.5$ \\
\hline $\bar{\nu}_{\mu} \mathrm{N} \rightarrow \mu^{+} \mathrm{h}^{ \pm}$ & $-(0.44 \pm 0.09)$ & $-(0.24 \pm 0.03)$ & $-(0.14 \pm 0.02)$ \\
Prediction [6] & -0.39 & -0.25 & -0.15 \\
\hline Neutrinos & $\langle Q\rangle^{\nu}$ & $\left\langle Q_{\mathrm{w}}\right\rangle^{\nu} r=0.2$ & $\left\langle Q_{\mathrm{w}}\right\rangle^{\nu} r=0.5$ \\
\hline${ }_{\mu} \mathrm{N} \rightarrow \mu^{-} \mathrm{h}^{ \pm}$ & $0.54 \pm 0.12$ & $0.42 \pm 0.04$ & $0.27 \pm 0.03$ \\
Prediction [6] & 0.60 & 0.39 & 0.26 \\
\hline
\end{tabular}

The average weighted charge results are given for two different values of the weight $r, r=0.2$ and $r=0.5$. Predictions from ref. [6] are also shown.

table 2. No significant changes in these measured quantities are observed indicating a negligible contamination from nuclear break-up products.

In terms of the quark-parton picture, any Bjorken $x$ dependence of the jet net charge, or the average weighted charge, at fixed c.m. energy, would reflect contributions from the $\overline{\mathrm{u}}$-quark jets in antineutrino-nucleon charged current interactions. In fig. 12 , we present the jet net charge and the average weighted charge $(r=0.5)$ with the selection $W>4 \mathrm{GeV}$ as a function of $x_{\mathrm{B}}$. Using the sea quark density distribution obtained from our data, we have calculated the predicted $x_{B}$ dependence of the jet net charge (average weighted charge) using the net charge (average weighted charge) values of -0.39 and $-0.60(-0.15$ and -0.26$)$ for the d-quark and $\bar{u}$-quark jets, respectively [6]. We then correct these predictions for the overlap between the target and current fragmentation regions by using the charge extrapolation result and the kinematical relation between $x_{\mathrm{B}},-q^{2}$ and $W^{2}\left(W^{2}=-q^{2}\left(1 / x_{\mathrm{B}}-1\right)+\right.$ $M^{2}$ ), and obtain the qualitative agreement with the experimental data (fig. 12). In

TABLE 2

Average net charge $\langle Q\rangle^{\bar{\nu}}$ and average weighted charge $\left\langle Q_{\mathrm{w}}\right\rangle^{\bar{\nu}}$ of the hadrons traveling forward in the hadronic c.m.s. in antineutrino charged current interactions for different total visible hadronic charge $Q_{\mathrm{V}}$ selections in an event

\begin{tabular}{ccccc}
\hline & \multicolumn{2}{c}{ Average energy correction } & \multicolumn{2}{c}{ Event-by-event energy correction } \\
& No. $Q_{\mathrm{V}}$ sel. & $-2 \leqslant Q_{\mathrm{V}} \leqslant 1$ & No. $Q_{\mathrm{V}}$ sel. & $-2 \leqslant Q_{\mathrm{V}} \leqslant 1$ \\
\hline$\langle Q\rangle^{\bar{\nu}}$ & $-(0.42 \pm 0.08)$ & $-(0.44 \pm 0.09)$ & $-(0.44 \pm 0.08)$ & $-(0.40 \pm 0.09)$ \\
$\left\langle Q_{\mathrm{w}}\right\rangle^{\bar{\nu}}$ & $-(0.20 \pm 0.03)$ & $-(0.24 \pm 0.04)$ & $-(0.22 \pm 0.03)$ & $-(0.25 \pm 0.04)$ \\
\hline
\end{tabular}

Results obtained with two independent energy reconstruction procedures are shown. 

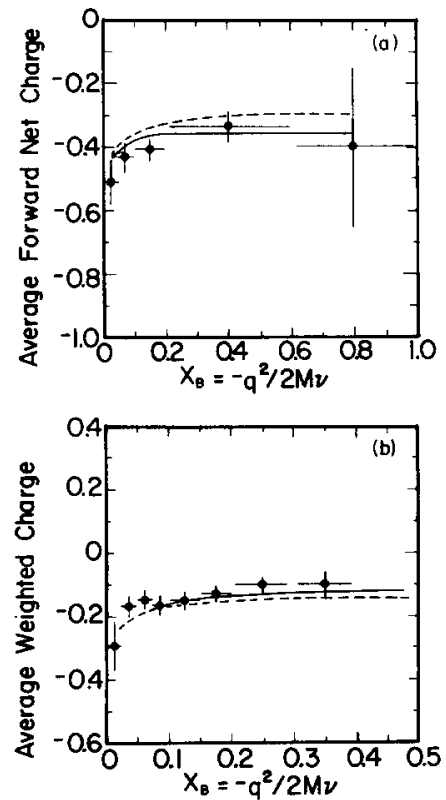

Fig. 12. (a) Average net charge and (b) average weighted charge $(r=0.5)$ of the antineutrino charged current induced jets as a function of Bjorken $x, x_{\mathrm{B}}=-q^{2} / 2 M \nu$. The dashed line represents the prediction described in the text. The solid line represents the prediction corrected for the overlap.
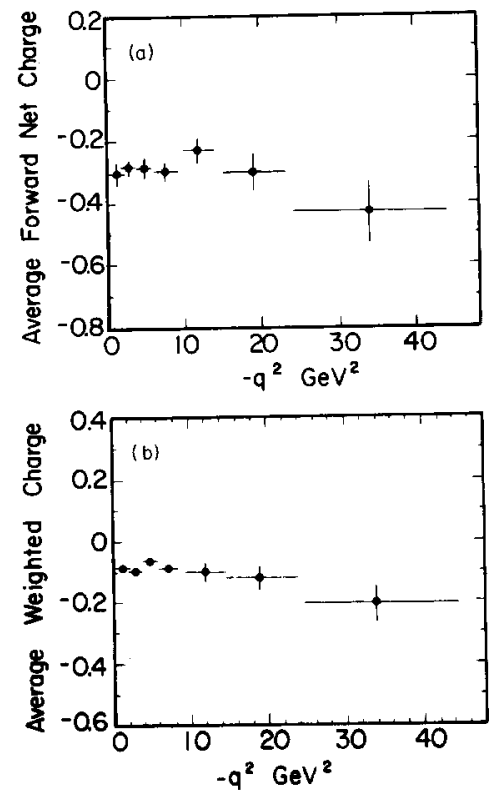

Fig. 13. (a) Average net charge and (b) average weighted charge $(r=0.5)$ of the antineutrino charged current induced jets as a function of $-q^{2}$. 
fig. 13a we have plotted the average jet net charge as a function of $-q^{2}$, and in fig. $13 \mathrm{~b}$ the average weighted charge $(r=0.5)$ as a function of $-q^{2}$ for the antineutrino charged current induced hadron jets. In these two figures, no $x_{\mathrm{B}}$ cut is applied. A decrease of the average jet net charge at high $-q^{2}$. would again indicate contributions from the $\overline{\mathrm{u}}$-quark jets. A qualitative agreement with this expectation is observed.

\section{Factorization test}

In the quark-parton model of deep inelastic scattering, the inclusive one-particle cross section is written as a product of the nucleon structure functions, $f_{i}\left(x_{\mathrm{B}}\right)$ and the quark-parton $\left(p_{i}\right)$ fragmentation functions, $D_{p_{i}}^{\mathrm{h}}(z)$, to a hadron h of fractional momentum $z$, i.e., at the limit $-q^{2} \rightarrow \infty$

$$
\frac{\mathrm{d}^{2} \sigma}{\mathrm{d} x \mathrm{~d} z}=\frac{G^{2} M E}{\pi} \sum_{i=1}^{n_{\mathrm{f}}} f_{i}\left(x_{\mathrm{B}}\right) D_{p_{i}}^{\mathrm{h}}(z),
$$

where $n_{\mathrm{f}}$ is the number of quark flavours (factorization hypothesis) [5].

In the following we will check the validity of the factorization hypothesis in our antineutrino-nucleon charged current interactions. The advantage of using antineutrino (neutrino) data is that in antineutrino (neutrino) induced charged current interactions one effectively selects the initial fragmenting quark flavour to be down (up). Thus, the quark-by-quark factorization hypothesis applies to the whole cross section. Any residual $x_{\mathrm{B}}$ dependence in the fragmentation function $D_{p}^{\mathrm{h}}(z)$ would violate this hypothesis.

We define the fragmentation function $D_{p}^{\mathrm{h}}$ within a given $-q_{0}^{2}$ interval in different intervals of $x_{\mathrm{B}}, x_{\mathrm{B} i}$, as follows:

$$
D_{p}^{\mathrm{h}}\left(z,-q_{0}^{2}, x_{\mathrm{B} i}\right)=\left.\frac{1}{N_{\mathrm{ev}}} \frac{\mathrm{d} N^{\text {tracks }}}{\mathrm{d} z}\right|_{x_{\mathbf{B} i},-q_{0}^{2}},
$$

where $N_{\text {ev }}$ and $N^{\text {tracks }}$ denotes the number of events and tracks, respectively. The ratio

$$
R=D_{p}^{\mathrm{h}}\left(z,-q_{0}^{2}, x_{\mathrm{B} i}\right) / D_{p}^{\mathrm{h}}\left(z,-q_{0}^{2}, x_{\mathrm{B} j}\right),
$$

where $i \neq j$, should then show deviation from a constant value if the factorization hypothesis is violated.

The inclusive one-particle distributions in the current fragmentation region are corrected for the energy smearing by our Monte Carlo model described earlier by calculating the energy desmearing functions, i.e., the ratios $(\mathrm{d} N / \mathrm{d} z)^{\text {unsmeared }}$ 
$(\mathrm{d} N / \mathrm{d} z)^{\text {smeared }}$. The correction varies between $5 \%$ at small $z$ values and $15 \%$ at large $z$. The energy desmearing functions, as calculated by the Monte Carlo program, for the ratios $R$ in different $x_{\mathrm{B}}$ regions are shown in fig. 14 .

Protons having momentum greater than $1 \mathrm{GeV} / c$ are not identified; they are treated as pions. Presence of these misidentified protons, due to the Lorentz transformation to the hadron c.m.s., causes a shift of the inclusive c.m.s. spectrum towards the forward c.m.s. hemisphere. We have investigated effects of these misidentified protons in the fractional energy distributions of the positively charged hadrons by using the lambda-hyperons observed in this experiment [27]. The total contribution from the protons in the current fragmentation region was found to be $(15 \pm 3) \%$. We emphasize that the selection $W>3 \mathrm{GeV}$ is applied to minimize the overlap between the target and current fragments and to suppress the quasi-elastic channels which generate $-q^{2}$ and $x_{\mathrm{B}}$ dependences.

Fig. 15a shows ratio $R$ plotted with $3<-q_{0}^{2}<10 \mathrm{GeV}^{2} / c^{2}$ for average values of $x_{\mathrm{B}}:\left\langle x_{\mathrm{B} 1}\right\rangle=0.1\left(0.01<x_{\mathrm{B} 1}<0.20\right)$ and $\left\langle x_{\mathrm{B} 2}\right\rangle=0.2\left(0.1<x_{\mathrm{B} 2}<0.3\right)$. No factorization violation is observed in the region where one can safely speak about the current fragments $(z>0.2)$. The factorization property of the inclusive one-particle cross section is further demonstrated in figs. $15 \mathrm{~b}$ and $\mathrm{c}$, where different average $x_{\mathrm{B}}$ values are chosen. Our results for the one-particle distributions show no significant $x_{\mathrm{B}}$ dependence.

Recent data for the moments of the non-singlet fragmentation functions $D_{p}^{\mathrm{NS}}=$ $D_{p}^{\mathrm{h}+}-D_{p}^{\mathrm{h}-}$, in a neutrino-proton experiment are claimed to show perturbative

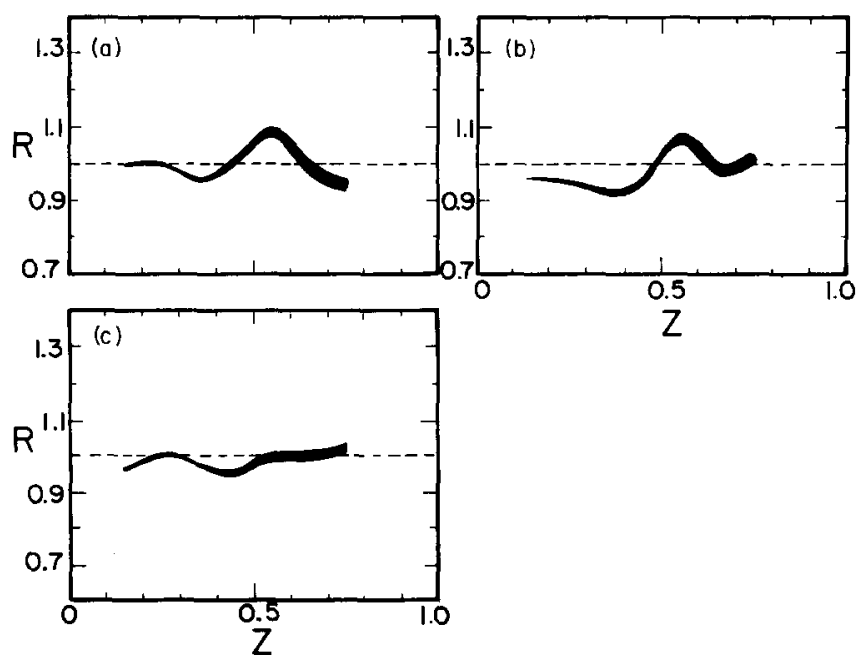

Fig. 14. Desmearing functions for the ratios $R$ (defined in the text) between the fragmentation functions evaluated in different Bjorken $x$ intervals: (a) $0.01<x_{\mathrm{B} 1}<0.20,0.1<x_{\mathrm{B} 2}<0.3$, (b) $0.01<x_{\mathrm{B} 1}<0.20$, $x_{\mathrm{B} 2}>0.2$, and (c) $0.1<x_{\mathrm{B} 1}<0.3, x_{\mathrm{B} 2}>0.2$. The selection in $-q^{2}$ is for $\mathrm{a}, \mathrm{b}$ and $\mathrm{c}, 3<-q^{2}<10$ $\mathrm{GeV}^{2} / c^{2}$. 

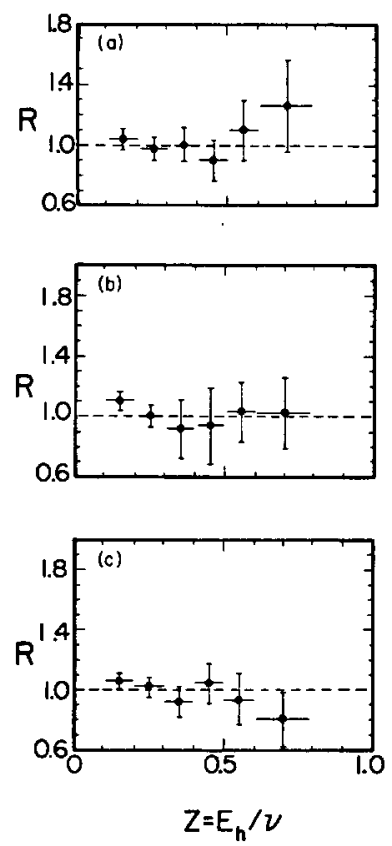

Fig. 15. Experimental results for the ratios $R$ (defined in the text) between the fragmentation functions evaluated in different Bjorken $x$ intervals: (a) $0.01<x_{\mathrm{B} 1}<0.20,0.1<x_{\mathrm{B} 2}<0.3$, (b) $0.01<x_{\mathrm{B} 1}<0.2$, $x_{\mathrm{B} 2}>0.2$, and (c) $0.1<x_{\mathrm{B} 1}<0.3, x_{\mathrm{B} 2}>0.2$. The selection in $-q^{2}$ is for $\mathrm{a}, \mathrm{b}$, and $\mathrm{c}, 3<-q^{2}<10$ $\mathrm{GeV}^{2} / c^{2}$.

QCD predicted factorization violation already at presently available neutrino energies [13]. However, our jet net charge results, which are related to $D^{\mathrm{NS}}$ functions by $\langle Q\rangle=\Sigma_{\mathrm{h}} e_{\mathrm{h}} \int \mathrm{d} z\left(D_{p}^{\mathrm{h}+}-D_{p}^{\mathrm{h}-}\right)=\Sigma_{\mathrm{h}} e_{\mathrm{h}} \int \mathrm{d} z D_{p}^{\mathrm{NS}}(z)$, where $e_{\mathrm{h}}$ is the (integer) charge of hadron $h$ in the jet, show the quark-parton model predicted $x_{\mathrm{B}}$ and $-q^{2}$ dependences and do not show perturbative QCD effects. We remark that the moment method results are effected by energy smearing which causes maximum uncertainty at high $z$ which are strongly weighed by the higher $z$ moments.

\section{Summary}

In agreement with the quark fragmentation picture, we have found evidence for d-quark jets (u-quark jets) in antineutrino (neutrino) charged current interactions. The probability of finding a $u$ - or d-quark in the quark jet cascade was measured to be $0.44 \pm 0.05$. No significant $x_{\mathrm{B}}$ dependence was seen in the single-particle distributions in the antineutrino induced jets.

We thank J.D. Bjorken, A. Buras, R.P. Feynman, R.D. Field and H.I. Miettinen for valuable discussions. 


\section{References}

[1] J.P. Berge et al., Phys. Lett. 91B (1980) 311

[2] Ch. Berger, H. Newman, G. Wolf and S. Orito, Proc. 1979 Int. Symp. on Lepton and photon induced interactions at high energy, ed. T.B. Kirk and H.D.I. Abarbanel

[3] M. Jacob, Phys. Scripta 19 (1979) 69

[4] J.D. Bjorken, Hadron final states in deep inelastic processes, 7th Int. Summer Inst. on Theoretical particle physics Hamburg (1975) p. 93, and references therein

[5] R.P. Feynman, Photon-hadron interactions (Benjamin, New York, 1972)

[6] R.D. Field and R.P. Feynman, Nucl. Phys. B136 (1978) 1

[7] J. Bell et al., Phys. Rev. D19 (1979) 1

[8] F.A. Nezrick, Nucl. Sci., vol. NS-22, no. 3 (1975) 1479

[9] R. Stefanski and H.B. White, Fermilab report TM-626A (1976), unpublished

[10] R.J. Cence et al., Nucl. Instr. 138 (1976) 245

[11] D. Sinclair, University of Michigan Research Note UMBC 78-3, (1978), unpublished

[12] G. Myatt, CERN/ECFA 72-4 (1972) 11

[13] J. Blietschau et al., Phys. Lett. 87B (1979) 281

[14] J.D. Bjorken, Phys. Rev. D7 (1973) 282

[15] N. Schmitz, Proc. 1979 Int. Symp. on Lepton and photon interactions at high energy, ed. T.B. Kirk and H.D.I. Abarbanel, p. 359

[16] D.H. Perkins, Lepton scattering and QCD, Lectures presented at Rutherford Laboratory Christmas Theoretical Physics Meeting, Rutherford, 1978

[17] G.R. Farrar and J.L. Rosner, Phys. Rev. D7 (1973) 2747

[18] R.N. Cahn and E.W. Colglazier, Phys, Rev. D9 (1977) 2658

[19] S.J. Brodsky and N. Weiss, Phys. Rev. D16 (1977) 2325

[20] P. Hoyer, C.-H. Lai and J.L. Petersen, Multiperipheral quark fragmentation, NORDITA-79/22 (1979)

[21] J. Whitmore et al., Phys. Rev. D16 (1977) 3137

[22] V. Ammosov et al., Phys. Lett. 93B (1980) 210

[23] J. Singh et al., Nucl. Phys. B140 (1978) 189;

R. Johnson et al., Phys. Rev. D17 (1978) 1292

[24] G.J. Feldman and M.L. Perl, SLAC preprint SLAC-Pub-72 (1977)

[25] R. Erickson et al., Phys. Rev. Lett. 42 (1979) 822

[26] I. Cohen et al., Phys. Rev. Lett. 40 (1978) 1614

[27] V. Ammosov et al., Nucl. Phys. Bl62 (1980) 205 